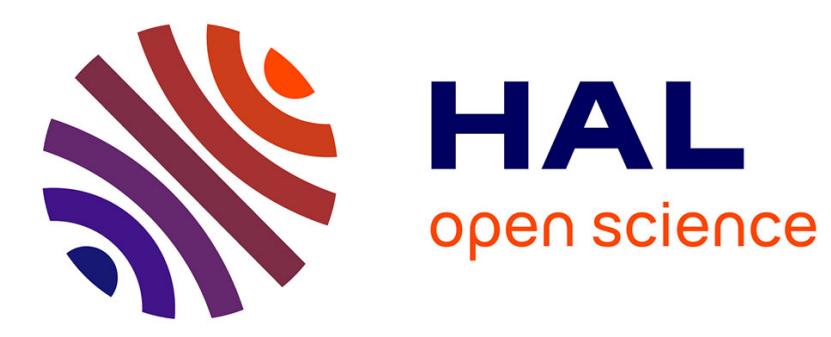

\title{
Évaluation et identification perceptives d'accents ouest-africains en français
}

Philippe Boula de Mareüil, Béatrice Akissi Boutin

\section{To cite this version:}

Philippe Boula de Mareüil, Béatrice Akissi Boutin. Évaluation et identification perceptives d'accents ouest-africains en français. Journal of French Language Studies, 2011, 21 (3), pp.361-379. 10.1017/S0959269510000621. hal-01411677

\section{HAL Id: hal-01411677 \\ https://auf.hal.science/hal-01411677}

Submitted on 7 Dec 2016

HAL is a multi-disciplinary open access archive for the deposit and dissemination of scientific research documents, whether they are published or not. The documents may come from teaching and research institutions in France or abroad, or from public or private research centers.
L'archive ouverte pluridisciplinaire HAL, est destinée au dépôt et à la diffusion de documents scientifiques de niveau recherche, publiés ou non, émanant des établissements d'enseignement et de recherche français ou étrangers, des laboratoires publics ou privés.

\section{(ㅇ)(1) $\$$}

Distributed under a Creative Commons Attribution - NonCommercial - NoDerivatives| 4.0 


\title{
Évaluation et identification perceptives d'accents ouest-africains en français
}

\author{
PHILIPPE BOULA DE MAREÜIL ${ }^{\mathrm{I}}$, \\ BÉATRICE AKISSI BOUTIN ${ }^{2}$ \\ ${ }^{\mathrm{I}}$ LIMSI-CNRS, Orsay \\ ${ }^{2}$ CLLE-ERSS, Toulouse et ILA, Abidjan
}

(Received January 20I0; revised July 20I0)

\begin{abstract}
RÉSUMÉ
Cette étude examine avec quel degré de granularité divers accents ouest-africains en français peuvent être distingués. Elle vise avant tout à déterminer si, en perception, des auditeurs de l'Afrique de l'Ouest sont capables d'identifier l'appartenance ethnolinguistique, le pays de résidence et le niveau d'études de locuteurs akan, bambara, sénoufo, mossi (de langue mooré) et wolof, enregistrés en Côte d'Ivoire, au Mali, au Burkina Faso et au Sénégal. Une expérience perceptive a été menée, d'où il ressort que les dimensions étudiées sont bien identifiées par les sujets. Pour les jeunes locuteurs comme pour les locuteurs plus âgés, en lecture comme en parole spontanée, les résultats sont très robustes. Ils sont de plus assez fidèles à la conscience linguistique auto-évaluée par les auditeurs : quand ceux-ci se déclaraient confiants pour reconnaître les accents en présence, tel a effectivement été le cas. Quelques indices acoustiques différenciant notamment les accents wolof (Sénégal) et akan (Côte d'Ivoire) ont finalement été dégagés.
\end{abstract}

\section{INTRODUCTION}

En Afrique comme en France, on peut entendre parler un «français africain » qui differe en partie du français standard. Une forme commune d' « accent africain » émerge-t-elle, quand bien même les locuteurs auraient des langues premières et des environnements linguistiques distincts? Comment les différences phonétiques sont-elles exploitées en perception, pour identifier des variétés de français parlées en Afrique? Ces questions ont été au cœur d'études à base de tests perceptifs centrés sur le Sénégal (Moreau, 200o) et le Mali (Lyche et Skattum, 20Io). Le présent travail s'inscrit dans la même lignée, impliquant ces pays et d'autres de l'Afrique de l'Ouest. Il se propose de même de mettre à l'épreuve certains discours épilinguistiques, lesquels peuvent être abordés sous trois angles.

Sous l'angle de la dialectologie perceptive qui s'est développée dans le sillage de Preston (I989), on cherche en l'absence d'input linguistique à cerner les représentations collectives et le savoir métalinguistique partagés par une communauté, en lien notamment avec la délimitation de variétés de langue dans 
notre cartographie mentale (Canut, I996, I997; Scherfer, 2000; Kuiper, 2005). Sous un autre angle socioculturel, on peut également étudier les attitudes évaluatives ou affectives face à des variétés de langues, exprimées en réaction à des stimuli linguistiques (Lambert, I972; Lafontaine, I986). Sous un angle plus cognitif enfin, on peut étudier l'aptitude à discerner différentes variétés de langues à partir d'un input phonétique (Clopper et Bradlow, 2009). En français, la perception comme compétence à appréhender la réalité linguistique avec une certaine justesse a été beaucoup moins évaluée - nous reviendrons sur quelques rares exceptions (Bauvois, I996; Armstrong et Boughton, I997; Moreau, 2000; Boughton, 2006; Boula de Mareüil et al., 2008; Woehrling, 2009; Lyche et Skattum, 20 Io). C'est cette faculté cognitive, dépendant également des habitudes du groupe, des croyances et stéréotypes sur la langue et ses variétés, que la présente étude vise à approcher, par des tests perceptifs d'identification d'accents. Savoir identifier des accents et savoir associer à chacun une constellation de traits linguistiques sont deux choses différentes. Ce dernier aspect est brièvement abordé ici; en revanche, dans l'approche expérimentale qui est développée, nous n'avons pas demandé d'émettre des jugements de valeur: les attitudes linguistiques n'auront donc pas ou que peu de place dans ce qui suit.

Afin de mettre en évidence les similitudes et les différences entre variétés de français ouest-africains, une expérience perceptive a été menée sur des échantillons de parole enregistrés au Burkina Faso, en Côte d'Ivoire, au Mali et au Sénégal dans le cadre du projet « Phonologie du Français Contemporain» $\left(\mathrm{PFC}^{1}\right)$ (Durand et al., 2002, 2005). Ces points d'enquête représentent au total une cinquantaine d'heures de parole (lue et spontanée), provenant de 52 locuteurs de différents groupes ethniques. Pour l'étude rapportée ici, les locuteurs appréciés, parlant français, étaient Akan (de langues baoulé, ébrié, alladian), Bambara, Sénoufo, Mossi (de langue mooré) ou Wolof. Des auditeurs originaires d'Afrique de l'Ouest sont-ils capables d'identifier ces différents accents? Avec quelle granularité des accents ouest-africains peuvent-ils être distingués? Si pour une oreille non-familière, retenant uniquement quelques invariants, on peut compter une seule étiquette «français d'Afrique subsaharienne », combien d'accents est-on en mesure de discerner quand on y est suffisamment exposé? Telles sont quelques questions que nous nous proposons d'aborder dans cette étude, qui peut trouver des applications directes.

Il est intéressant de mettre en relation les connaissances/représentations linguistiques des sujets, qui s'élaborent socialement, avec leurs performances perceptives individuelles. Plusieurs opérations de catégorisation s'entrecroisent: ainsi, la variation du français selon le niveau d'études, souvent mise en avant dans

${ }^{1}$ http://www. projet-pfc.net Nous remercions chaleureusement Jacques Durand, Bernard Laks et Chantal Lyche, responsables de ce vaste projet qu'est PFC. Nous exprimons notre plus profonde gratitude à Ingse Skattum, Gisèle Prignitz, Gabriel-Marie Gueye et Mame Thierno Cissé pour les données enregistrées, ainsi qu'à tous les sujets que nous avons sollicités pour les tests perceptifs. Enfin, notre reconnaissance va à Nigel Armstrong et aux relecteurs qui ont eu entre les mains une version antérieure du présent article: leurs commentaires constructifs nous ont été bien utiles. 
les descriptions du français en Afrique (Knutsen, 2007; Lyche et Skattum, 20Io), est ici mise à l'épreuve (les locuteurs pouvant être de niveaux d'études assez variés). La catégorie sociale est-elle plus perceptible que l'appartenance à une ethnie ou à un pays? Nous tenterons de le quantifier à travers différentes tâches. De même est appréciée l'émergence d'accents nationaux, au-delà des accents régionaux ou ethniques.

Après une présentation très générale du contexte ouest-africain, nous décrirons le protocole expérimental, le matériel, les locuteurs et les auditeurs. Nous donnerons ensuite les résultats, que nous discuterons en ouvrant quelques pistes relatives aux indices acoustiques sur lesquels ont pu s'appuyer les sujets.

\section{CONTEXTE}

La plupart des pays africains sont plurilingues, notamment ceux dits francophones, où le français, langue exogène, est langue officielle, langue de l'administration et langue d'enseignement. La plupart des locuteurs ouest-africains francophones n'ont qu'une pratique non-native et non-usuelle du français, qui passe par l'école, alors que le taux d'alphabétisation peut être très faible (Lyche et Skattum, 20Io). On estime en revanche qu'un quart au moins des Ivoiriens ont acquis le français dans la petite enfance ou en dehors de l'école (Boutin et Turcsan, 2009): phénomène atypique, cette «nativisation » du français en l'absence d'une langue africaine majoritaire en vient à constituer un trait identitaire de la Côte d'Ivoire. La situation d'un français parlé comme langue première ne se retrouve dans les pays limitrophes que dans des groupes minoritaires. Par la prononciation (et la syntaxe de surcroit), les formes de français vernaculaire ivoirien présentent un certain nombre de différences par rapport au français de France, qui peut être perçu comme «maniéré »: les écarts entre formes exogènes et locales de français tendent à devenir des marqueurs de l'appartenance à la communauté ivoirienne (Ploog, 2002; Boutin et Turcsan, 2009, inter alia). Le Sénégal, avec le rôle essentiel joué par la langue wolof, qui symbolise l'appartenance à la culture sénégalaise, représente le cas opposé (Boutin et Gueye, à paraitre). Malgré ses trois siècles de présence, le français n'est ni véhiculaire ni vernaculaire: il reste pour les locuteurs une langue étrangère, dans laquelle l'enseignement est pourtant dispensé et le discours officiel délivré.

Parmi les langues africaines parlées en Afrique de l'Ouest, les langues akan, le bambara, le mooré et le wolof (toutes de la branche Niger-Congo) constituent des langues majeures de la sous-région. Le terme akan - moins englobant mais plus courant que celui de kwa (Tymian et al., 2003) - désigne un groupe de peuples au Ghana ainsi qu'au sud-est et au centre de la Côte d'Ivoire. Les langues ivoiriennes du groupe kwa sont, entre autres, l'agni, le baoulé, l'alladian, l'ébrié. Le bambara (du groupe mandé), principalement parlé au Mali, est la langue la plus couramment comprise de ce pays: il est la langue vernaculaire de $40 \%$ de la population et langue véhiculaire pour encore $40 \%$ de la population. Le bambara est parlé aussi au Burkina Faso et en Côte d'Ivoire (éventuellement sous sa forme véhiculaire de dioula). Le mooré (du groupe gur) est la langue des Mossi, ethnie majoritaire et historiquement 
dominante au Burkina Faso. Il est comme le jula (dioula) langue véhiculaire dans ce pays. Le wolof (du groupe atlantique) est la principale langue parlée au Sénégal : langue des Wolof, il est en pleine expansion et est actuellement parlé par plus des trois quarts des Sénégalais (Cissé, 2005). D’autres langues, comme celles des Sénoufo, parlées au nord de la Côte d'Ivoire, au sud-est du Mali et au sud-ouest du Burkina Faso, ne constituent pas des langues majeures. Alors que les langues sénoufo font partie du même groupe gur que le mooré, leurs locuteurs parlent en général également bambara (ou dioula), et se rapprochent culturellement davantage des Bambara que des Mossi. Les locuteurs des langues sénoufo présentent donc un autre intérêt.

Toutes ces langues ont des systèmes phonologiques (et syntaxiques) très différents. Le wolof se distingue peut-être plus encore par l'absence de tons lexicaux, alors que les autres langues sont tonales: le wolof est une langue à accent fixe qui porte sur la première syllabe du mot. On peut dès lors se demander si le français parlé par les Wolof s'oppose aussi aux autres formes de français ouest-africain. Il y a là des hypothèses linguistiques intéressantes à tester, sur lesquelles peu d'études ont porté. Mais il s'agit avant tout de vérifier si des auditeurs ouest-africains sont à même de distinguer les différents accents.

\section{EXPÉRIENCE：TÂCHE ET PROTOCOLE}

Une expérience perceptive a été conduite pour déterminer comment des auditeurs ouest-africains évaluent et identifient les accents étudiés. Il était demandé aux sujets, après quelques renseignements à caractère autobiographique (âge, niveau d'études, etc.) d'indiquer quelle était leur familiarité avec les différents accents: les sujets devaient spécifier si oui/non ils se sentaient capables de reconnaitre tel ou tel accent parmi les cinq proposés quand une personne parle français. Puis, lors d'une brève phase de familiarisation, ils écoutaient des échantillons de parole provenant de locuteurs (non utilisés par la suite) dont le pays était précisé: Burkina Faso, Côte d'Ivoire, Mali ou Sénégal. Le test proprement dit consistait ensuite à écouter 40 extraits sonores et, pour chacun, à accomplir deux types de tâches:

- évaluer le degré d'accent du locuteur ou de la locutrice sur une échelle continue graduée de o à 5 ;

- évaluer son niveau d'études sur une autre échelle continue graduée de o à 5;

- identifier son appartenance ethnolinguistique (akan, bambara, sénoufo, mooré ou wolof);

- identifier son pays de résidence (Burkina Faso, Côte d'Ivoire, Mali ou Sénégal).

L'étude, soulignons-le, s'attachait à la conceptualisation de la variation sociolinguistique (relier un accent avec un groupe/type de locuteurs) mais ne laissait pas de place à l'évaluation sur des échelles de valeurs ni aux affects concernant les variétés de langue. Quant à notre choix de demander aux sujets une évaluation du niveau d'études, il est en partie la conséquence de la difficulté à faire identifier une catégorie socioprofessionnelle. La difficulté de ce genre de tâche en contexte 
occidental est accrue en contexte africain, les sociétés étant structurées de façon différente.

Une interface $w^{2} b^{2}$ permettait de lire les instructions, écouter les stimuli et saisir les réponses. Elle proposait deux curseurs à déplacer pour les tâches d'évaluation et deux choix forcés à effectuer en cliquant sur des boutons à cocher - parmi 5 appartenances ethnolinguistiques et 4 pays — pour les tâches d'identification. Les degrés d'accent étaient paraphrasés comme suit: (O) pas d'accent, (I) petit accent, (2) accent modéré, (3) accent plutôt fort, (4) fort accent, (5) très fort accent. Les niveaux d'études l'étaient de la façon suivante: (O-I) primaire, (I-2) collège, (2-3) lycée, (3-4) début d'enseignement supérieur, $(4-5)$ au moins bac +3 .

Les stimuli étaient présentés dans un ordre aléatoire qui changeait pour chaque auditeur. Cette précaution nous a semblé d'autant plus importante que, pour l'évaluation du degré d'accent sur une échelle relative notamment, les sujets n'avaient d'autre point de repère que l'étape de familiarisation et leur propre expérience quotidienne. Chaque extrait pouvait être écouté autant de fois que cela était jugé nécessaire. Une fois passé à un autre stimulus, il n'était cependant plus possible de revenir à des stimuli précédents.

À la fin du test, les sujets étaient invités à apporter librement des commentaires sur les indices les plus saillants qui avaient guidé leurs décisions. Ils devaient également préciser s'ils pensaient avoir répondu au hasard pour une identification particulière. Ces commentaires, demandés à la fin du test et non au fil de l'écoute des séquences sonores, pouvaient se rapporter à des identités erronées puisque les sujets n'avaient pas de retour sur leurs réponses.

\section{LOCUTEURS ET STIMULI}

Les stimuli retenus pour le test perceptif provenaient de 20 locuteurs (I I hommes, 9 femmes, âgés de 47 ans en moyenne), de milieux socioprofessionnels variés. Les locuteurs - 4 par groupe ethnolinguistique (akan, bambara, sénoufo, mooré et wolof) - ont été enregistrés sur leur lieu de résidence ou dans un endroit calme, en milieu urbain, à Abidjan, Bamako, Ouagadougou et Dakar, capitales économiques propices aux échanges en français. Disons toutefois ici qu'une contrainte était imposée par le projet PFC, pour pouvoir appliquer un protocole labovien (Labov, 1972): les locuteurs devaient savoir à la fois lire et converser librement en français. Leur répartition par pays, tranche d'âge et niveau d'études est donnée schématiquement dans la figure I. Dans notre corpus expérimental, tous les locuteurs wolof (au Nord-Ouest) étaient Sénégalais, tous les locuteurs akan étaient Ivoiriens (au Sud), tous les locuteurs du mooré étaient Burkinabè. Les locuteurs sénoufo étaient ressortissants de trois pays (Mali, Burkina Faso et Côte d'Ivoire) et les locuteurs bambara ressortissants de deux pays (Mali et Côte d'Ivoire). Parmi les Sénoufo, le locuteur burkinabè avait fait un long séjour en Côte d'Ivoire, et le

${ }^{2}$ http://www.audiosurf.org/test_perceptif_africa/ Les échantillons de parole utilisés peuvent être écoutés à cette adresse. 


\begin{tabular}{|c|c|c|c|c|c|c|c|c|c|c|c|}
\hline \multirow{4}{*}{ MALI } & jB & \multicolumn{3}{|c|}{ wolof } & \multicolumn{2}{|c|}{ SÉNÉGAL } & & & & & \multirow{3}{*}{$\begin{array}{c}\text { BURKINA } \\
\text { FASO }\end{array}$} \\
\hline & & imbar & & & énou & & & nooré & & & \\
\hline & jA & vB & vD & vB & $\mathrm{vC}$ & $\mathrm{jE}$ & jA & jC & $\mathrm{vA}$ & $\mathrm{vE}$ & \\
\hline & & $\begin{array}{l}\text { TE } \\
\text { OIRE }\end{array}$ & $\mathrm{vD}$ & jD & $\mathrm{jB}$ & $\begin{array}{c}\text { akan } \\
\mathrm{jE}\end{array}$ & jE & $\mathrm{vC}$ & & & \\
\hline
\end{tabular}

Figure I. Répartition des locuteurs par groupes ethnolinguistiques (encadrés), par pays (Burkina Faso en hachuré /, Côte d'Ivoire en treillis foncé, Mali en hachuré \, Sénégal en treillis clair), tranche d'âge et niveau d'études.

locuteur ivoirien, dont le français était la langue première, avait toujours vécu à Abidjan. On avait en outre une locutrice ivoirienne bambara qui avait fait un long séjour au Mali dans son enfance.

Dans la figure I également, le niveau d'études est rapporté selon les 5 catégories distinguées ci-dessus: primaire (A), collège (B), lycée $(C)$, début d'enseignement supérieur (D), au moins bac +3 (E). De plus, les locuteurs sont divisés en deux groupes d'âge à partir de la moyenne de l'échantillon, considérant comme «jeunes » (noté j) les io locuteurs de moins de 47 ans (moyenne: 39 ans) et comme "vieux » (noté v) les io locuteurs de plus de 47 ans (moyenne: 57 ans). Ce seuil de 47 ans, que nous n'avions pas choisi, et la division « jeunes »/ «vieux » qui s'ensuit, correspondent peu ou prou à deux situations sociolinguistiques se distinguant par une naissance avant les Indépendances pour les « vieux » et après pour les «jeunes ». Pour le premier groupe, "l'école ancienne » était le lieu d'un apprentissage du français dont l'efficacité n'était pas remise en question (Boutin et Prignitz, 20IO).

Pour chaque locuteur, deux échantillons ont été sélectionnés: une phrase lue (de 30 mots) et un énoncé de parole spontanée d'une dizaine de secondes, comme dans des expériences antérieures (Boula de Mareüil et al., 2008). La phrase lue (la même pour tous les locuteurs) était: «Beaulieu préfere être inconnue et tranquille plutôt que de se trouver au centre d'une bataille politique dont, par la télévision, seraient témoins des millions d'électeurs. " L'énoncé de parole spontanée était extrait de conversations libres ou d'entretiens guidés, d'après les critères suivants: cohérence du propos, absence de références culturelles, spatiales ou socio-économiques, de traits lexicaux ou syntaxiques qui pouvaient être typiques d'une origine particulière. La parole spontanée évite que les auditeurs aient à écouter systématiquement la même phrase et reflète mieux la façon naturelle de parler. La lecture, de surcroît, permet des comparaisons toutes choses égales par ailleurs et garantit que les différences entre locuteurs ont trait à la prononciation.

\section{AUDITEURS}

Le test a été soumis à 20 auditeurs ouest-africains $(7$ hommes, I 3 femmes, âgés de 35 ans en moyenne), sans problèmes d'audition connus. Les sujets n'étaient 
pas payés pour leur participation, qui était en moyenne de 30 minutes. À une ou deux exceptions près, ils avaient tous passé la plus grande partie de leur vie en Côte d'Ivoire, et avaient un niveau d'étude d'au moins bac +3 . La moitié d'entre eux résidait à Abidjan, l'autre moitié dans d'autres villes de Côte d'Ivoire ou en France. La moitié d'entre eux avait le français pour langue maternelle, l'autre moitié des langues africaines comme le gouro (du groupe mandé sud, centre-ouest de la Côte d'Ivoire) ou le baoulé (du groupe kwa). Deux d'entre eux, résidant en France, étaient de langue wolof. Cet ensemble d'auditeurs, presque uniquement composé d'Ivoiriens, présente un certain intérêt: par sa position géographique et économique, la Côte d'Ivoire se trouve être un lieu de contacts et de migrations privilégié dans la région. La familiarisation avec différentes langues africaines et formes de français favorise la construction de connaissances partagées sur les langues et accents.

La majorité des sujets se disait capable, avant le test, de reconnaître les accents en présence, à l'exception de l'accent sénoufo en français. Ils n'étaient que 7 sur 20 à penser pouvoir identifier l'accent sénoufo, contre I 7 pour l'accent akan, I 2 pour l'accent bambara, I 5 pour l'accent mooré et 20 sur 20 pour l'accent wolof.

\section{RÉSUltats PERCEPTifs : TÂCHES D’évaluation}

Dans ce qui suit, nous allons étudier l'éventuel effet sur la perception des accents que peuvent avoir le niveau d'études, l'âge des locuteurs et le style de parole (lu ou spontané). Les résultats obtenus sont exprimés en termes de degré d'accent (entre o et 5 ), de niveau d'études perçu (entre o et 5) et de taux d'identification de ce niveau d'études par rapport aux 5 catégories (A, B, C, D, E) distinguées ci-dessus. Par exemple, pour un échantillon de parole provenant d'un locuteur de niveau $\mathrm{B}$, on considère que ce niveau est bien identifié si le niveau d'études perçu est supérieur ou égal à I et inférieur à 2 . En moyenne, les résultats sont très proches si on considère l'inégalité large pour la borne droite.

En moyenne, le degré d'accent des locuteurs $(3,0 / 5)$ est jugé comme plutôt fort, et le niveau d'études perçu $(2,7 / 5)$ correspond au bac. Estimée en termes d'identification correcte, le niveau d'études est bien reconnu à $33 \%$, ce qui est très significativement au dessus du hasard d'après un test de $\chi^{2}$. Le tableau I montre l'évolution de ces chiffres en fonction du niveau d'études réel des locuteurs. On voit que le degré d'accent perçu est relativement stable (au dessus de 3) hormis pour le groupe de locuteurs les plus diplômés (au moins bac +3 ), qui ont 2,3: on a donc globalement une diminution du degré d'accent perçu avec le niveau d'études. Le niveau d'études perçu, quant à lui, croît régulièrement, tandis que le taux d'identification de ce niveau d'études varie de $23 \%$ à $48 \%$, sans tendance particulière. En termes de catégories (A, B, C, D ou E), le niveau d'études est majoritairement bien perçu, sauf le niveau B (collège) perçu comme $\mathrm{C}$ (lycée).

Des analyses de variance (ANOVA) révèlent que l'effet du Niveau d'études réel $(\mathrm{A}, \mathrm{B}, \mathrm{C}, \mathrm{D}$ ou E) est significatif pour le degré d'accent $[F(4,795)=22 ; p<\mathrm{O}, \mathrm{OO} \mathrm{I}]$ 
Tableau I. Degré d'accent, niveau d'études perçus et taux d'identification correcte de ce niveau par rapport au niveau d'études réel.

\begin{tabular}{lllllll}
\hline \hline \multirow{2}{*}{ Niveau d'études } & primaire & $\begin{array}{l}\text { collège } \\
(\mathrm{A})\end{array}$ & $\begin{array}{l}\text { lycée } \\
(\mathrm{B})\end{array}$ & $\begin{array}{l}\text { déb. ens. } \\
\text { (C) }\end{array}$ & $\begin{array}{l}\geq \text { bac }+(\mathrm{D}) \\
3(\mathrm{E})\end{array}$ & moyenne \\
\hline degré d'accent $(/ 5)$ & 3,3 & 3,3 & $3, \mathrm{I}$ & $3, \mathrm{O}$ & 2,3 & $\mathbf{3}, \mathbf{0}$ \\
niveau perçu $(/ 5)$ & $\mathrm{I}, 7$ & 2,3 & 2,5 & $3, \mathrm{I}$ & 3,5 & $\mathbf{2 , 7}$ \\
id. correcte niveau $(\%)$ & 30 & 23 & 25 & 48 & 39 & $\mathbf{3 3}$ \\
\hline \hline
\end{tabular}

Tableau 2. Degré d'accent, niveau d'études perçus et taux d'identification correcte de ce niveau par rapport au niveau d'études réel selon l'âge des locuteurs et le style de parole.

\begin{tabular}{lllll}
\hline \hline & jeunes & vieux & lecture & spontané \\
\hline degré d'accent $(/ 5)$ & 3,0 & 2,9 & 3,0 & 2,9 \\
niveau perçu $(/ 5)$ & 2,8 & 2,7 & 2,8 & 2,6 \\
id. cor. niveau $(\%)$ & 32 & 34 & 33 & 33 \\
\hline \hline
\end{tabular}

et le niveau d'études perçu $[F(4,795)=7 \mathrm{I} ; p<$ o,00I $]$. Des tests de Student deux à deux (pairwise $t$-tests), cependant, montrent que l'effet n'est significatif sur le degré d'accent qu'avec les locuteurs les plus diplômés (de niveau E), tandis qu'il est pratiquement toujours significatif sur le niveau d'études perçu: la seule exception est la différence entre 2,3 (collège) et 2,5 (lycée). Pour les 800 réponses des auditeurs (40 stimuli $\times 20$ sujets), une corrélation a par ailleurs été calculée entre le degré d'accent et le niveau d'études perçus. Cette corrélation est faiblement négative $(-0,2)$, montrant par là que le lien n'est pas évident entre ces deux évaluations.

Les résultats des évaluations pour les «jeunes » et les «vieux » locuteurs, la lecture et la parole spontanée sont consignés dans le tableau 2. On observe que ces facteurs de l'âge et du style n'ont que très peu d'incidence sur les résultats. D'après des ANOVA, l'effet de l'Âge (jeune ou vieux) n'est jamais significatif; le Style (lu ou spontané) a uniquement un effet significatif sur le niveau d'études perçu $[F(\mathrm{I}, 798)=6,95 ; p<\mathrm{O}, \mathrm{OI}]$, l'interaction avec l'Âge restant marginale. La cohérence des résultats est intéressante à noter, car dans d'autres circonstances, en matière d'accents régionaux en français, le degré d'accent perçu a tendance à augmenter avec l'âge des locuteurs (Léon et Léon, I997; Sobotta, 2006; Boula de Mareüil et al., 2008; Woehrling, 2009). De plus, le niveau d'études n'a pas la même valeur selon l'âge des locuteurs. Dans nos données, les niveaux d'études étaient équilibrés dans les deux tranches d'âges: la moitié des jeunes comme des vieux locuteurs avait le bac. Quant à la lecture par rapport à la parole spontanée, on peut penser que de possibles difficultés à lire (qui peuvent aussi avoir pour cause un problème de vue) sont interprétées comme reflétant un niveau d'études bas. De fait, un locuteur de niveau A (primaire) avait manifestement, selon nous, du mal à lire (hésitations, reprises, erreurs); en moyenne calculée sur les réponses des 20 auditeurs, son niveau d'études a été évalué à 0,5 (i.e. primaire) en lecture et à I,5 (i.e. secondaire) en parole spontanée. Mais globalement, il semble que les particularités de la prononciation se retrouvent d'un style à l'autre. 


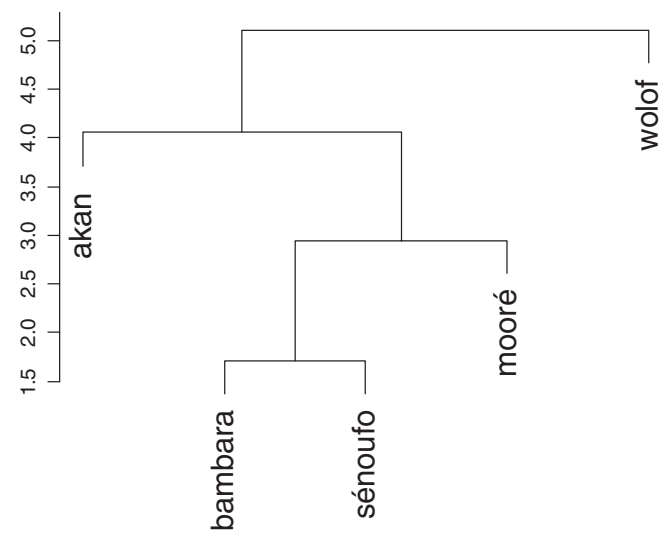

Figure 2. Dendrogramme représentant l'identification de l'appartenance ethnolinguistique.

La combinatoire est trop importante par rapport à nos données pour ventiler les résultats par groupe ethnolinguistique et par pays. Nous reviendrons ci-dessous sur le rôle du degré d'accent et du niveau d'études perçus en matière d'identification.

\section{RÉSULTATS PERCEPTIFS：TÂCHES D'IDENTIFICATION}

Les résultats des tâches d'identification sont rapportés dans les tableaux 3 et 4 pour l'appartenance ethnolinguistique et le pays respectivement. Une visualisation en est également donnée dans les figures 2 et 3 , comme nous allons le voir.

L'appartenance ethnolinguistique est correctement identifiée à $5 \mathrm{I} \%$, ce qui est très significativement mieux que le hasard $(20 \%)$ d'après un test de $\chi .^{2}$. Seul l'accent sénoufo est mal identifié: il a, davantage que l'étiquette correcte, reçu l'étiquette «bambara » et même l'étiquette «akan »- dans une proportion proche $(26 \%)$ de la confusion symétrique akan-sénoufo (22\%). L'accent bambara est lui-même correctement identifié dans plus de $50 \%$ des cas, de même que l'accent des locuteurs akan. L'accent wolof est reconnu à $8 \mathrm{I} \%$ et n'est ensuite confondu avec l'accent bambara que dans $7 \%$ des cas. L'accent mooré en français n'est bien identifié qu'à une majorité relative (46\%), mais on note qu'il n'a jamais reçu l'étiquette wolof.

Des techniques d'analyse de données permettent de représenter cette matrice de confusion de façon synthétique, sous la forme d'un dendrogramme ou d'un plan à deux dimensions. Le dendrogramme qui est représenté dans la figure 2 a été obtenu au moyen du logiciel $\mathrm{R}^{3}$, par un algorithme de clustering. Il montre bien, comme

${ }^{3}$ http://www.r-project.org Un algorithme agglomératif hiérarchique a été utilisé, avec une distance euclidienne entre les vecteurs constituant les lignes de la matrice de confusion. Un algorithme d'échelonnement multidimensionnel fondé sur une distance euclidienne a également été utilisé. 
Tableau 3. Matrice de confusion concernant l'appartenance ethnolinguistique (\%).

\begin{tabular}{lrllcc}
\hline \hline orig. \rép. & akan & bambara & sénoufo & mooré & wolof \\
\hline akan & $\mathbf{6 I}$ & IO & 22 & 6 & I \\
bambara & 6 & $\mathbf{5 2}$ & I I & 24 & 7 \\
sénoufo & 26 & $\mathbf{4 I}$ & I 6 & I 6 & I \\
mooré & I 4 & 2 I & I & $\mathbf{4 6}$ & O \\
wolof & 2 & I I & I & 6 & $\mathbf{8 I}$ \\
\hline \hline
\end{tabular}

Tableau 4. Matrice de confusion concernant le pays des locuteurs (\%).

\begin{tabular}{lcllc}
\hline \hline orig. \rép. & Burkina Faso & Côte d'Ivoire & Mali & Sénégal \\
\hline Burkina Faso & $\mathbf{4 I}$ & 36 & 23 & O \\
Côte d'Ivoire & 9 & $\mathbf{7 5}$ & I6 & I \\
Mali & I9 & I9 & $\mathbf{5 6}$ & 6 \\
Sénégal & 4 & 2 & IO & $\mathbf{8 3}$ \\
\hline \hline
\end{tabular}

le tableau 3, que l'accent wolof se détache des autres. Vient ensuite l'accent akan, les accents bambara et sénoufo étant regroupés au plus profond de l'arbre. En accord avec cette confusion bambara/sénoufo, I7 sujets sur 20 ont répondu positivement à la question « avez-vous le sentiment d'avoir répondu au hasard entre le bambara et le sénoufo? » qui leur était posée à la fin du test.

Une ANOVA a été menée sur les réponses comptées comme correctes (I) ou incorrectes (o) avec le facteur aléatoire Sujet et les deux facteurs intra-sujets Style (lu ou spontané) et Âge du locuteur (jeune ou vieux). Les facteurs Style et Âge n'ont pas d'effet significatif, même si les jeunes locuteurs sont légèrement mieux identifiés que les vieux (à 53\% contre 50\%) et que les extraits de lecture sont légèrement mieux identifiés que les extraits de parole spontanée (à $54 \%$ contre 49\%). L'interaction entre Style et Âge est également marginale.

Si l'on regarde les résultats stimulus par stimulus, 25 sur 40 sont correctement identifiés en termes d'appartenance ethnolinguistique par au moins la moitié des auditeurs. Tous les échantillons issus de locuteurs sénoufo sont mal identifiés, tandis que tous les échantillons issus de locuteurs wolof, que ce soit en lecture ou en parole spontanée, ont reçu l'étiquette «wolof » de la part d'au moins is auditeurs sur 20. Ces chiffres reflètent assez bien les résultats affichés dans le tableau 3 .

Le pays des locuteurs, parmi 4 , a été correctement identifié à $63 \%$, ce qui est très significativement mieux que le hasard d'après un test de $\chi^{2}$. Pour chaque pays, la réponse majoritaire est la bonne ( $c f$. tableau 4$)$, même si la majorité n'est que relative pour le Burkina Faso. Pour ce dernier pays, dont les locuteurs sélectionnés étaient essentiellement de langue mooré, on retrouve des patrons de réponses similaires à ceux qui portaient sur l'appartenance ethnolinguistique - aucune confusion, notamment, avec le Sénégal dont tous les locuteurs retenus étaient wolophones.

Un algorithme d'échelonnement multidimensionnel (scaling) a été utilisé pour représenter graphiquement une sorte de distance perceptive entre les différents pays. Le résultat du scaling obtenu pour les pays, au moyen du logiciel $\mathrm{R}^{3}$, est donné 


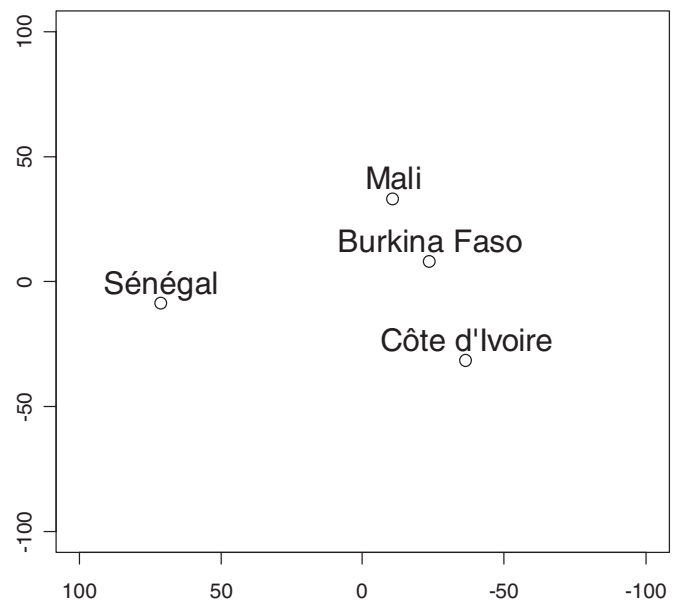

Figure 3. Plan à deux dimensions représentant l'identification par pays.

dans la figure 3: dans ce plan à deux dimensions, l'axe des ordonnées représente la première dimension et l'axe des abscisses, orienté de façon à faire figurer le Sénégal à l'Ouest, représente la deuxième dimension. On voit immédiatement que le Sénégal est isolé, alors qu'un continuum perceptif semble aller de la Côte d'Ivoire au Mali en passant par le Burkina Faso.

De nouveau, une ANOVA a été conduite sur les réponses comptées comme correctes (I) ou incorrectes (O) avec le facteur aléatoire Sujet et les deux facteurs intra-sujets Style (lu ou spontané) et Âge du locuteur (jeune ou vieux). L'effet du Style n'est pas significatif, même si le pays est légèrement mieux identifié sur la lecture (à 64\%) que sur la parole spontanée (à 62\%). L'effet de l'Âge des locuteurs est ici significatif $[F(\mathrm{I}, \mathrm{I} 9)=5,77 ; p<0,05]$ : le pays est significativement mieux identifié pour les jeunes locuteurs (à 68\%) que pour les vieux locuteurs (à $59 \%$ ). L'interaction Style $\times$ Âge n'est toujours pas significative. Sans tirer de conclusions hâtives sur un effet majeur de l'âge des locuteurs, ces résultats, sur lesquels nous reviendrons, sont intéressants dans la mesure où ce sont surtout les jeunes Ivoiriens qui sont bien identifiés (à $87 \%$ ). Le facteur intra-sujet Niveau d'études des locuteurs a de la même façon été analysé, restreint à «bac » ou " pas bac » par manque de données, pour des tests statistiques; cependant, il n'a pas ici d'effet significatif - le pays étant légèrement mieux reconnu pour les locuteurs ayant le bac (à $66 \%$ contre $60 \%)$.

Si l'on examine les résultats stimulus par stimulus, 3I sur 40 sont correctement identifiés en termes de pays par au moins la moitié des auditeurs. Les échantillons qui ne sont pas bien identifiés viennent essentiellement du Burkina Faso, ce qui est en accord avec la matrice de confusion du tableau 4.

Afin d'évaluer le lien entre identifications de l'appartenance ethnolinguistique et du pays, les 800 réponses des auditeurs ont été comptées comme correctes (I) 
ou incorrectes (o), et une corrélation a été calculée entre les deux séries de chiffres résultants. Avec un coefficient de corrélation de o,6, il y a bien un lien entre l'appartenance ethnolinguistique et le pays identifiés. Dans notre corpus, de fait, il y a une bijection wolof-Sénégal - et partant les wolophones ont été identifiés comme Sénégalais à 83\%. Dans plus de 99\% des cas, la réponse «wolof » (correcte ou non) était associée au Sénégal et la réponse « akan » à la Côte d'Ivoire; dans plus de $90 \%$ des cas la réponse «bambara » était associée au Mali, la réponse "mooré » au Burkina Faso, la réponse « sénoufo » à la Côte d'Ivoire.

Il peut être intéressant également de regarder trois cas particuliers de notre corpus: le locuteur burkinabè sénoufo (BS), le locuteur ivoirien sénoufo (IS) et la locutrice ivoirienne bambara (IB). BS a majoritairement été identifiée comme ivoirien bambara, IS comme ivoirien akan et IB comme malienne bambara. Des facteurs tels que la mobilité géographique et l'environnement linguistique peuvent expliquer, pour ces locuteurs, l'identification dont ils font l'objet ( $f f$. supra). On ne peut donc pas conclure, de ce seul examen, sur ce qui prime entre appartenances nationale et ethnolinguistique.

Tirant profit des résultats des tâches d'évaluation et d'identification, nous avons calculé, à partir des 800 séries de réponses des auditeurs, les corrélations entre d'une part l'identification de l'appartenance ethnolinguistique ou du pays comptée comme correcte (I) ou incorrecte (O) et d'autre part le degré d'accent ou le niveau d'études perçus. Les quatre coefficients de corrélation résultants sont égaux à o, I donc faibles.

\section{QUELQUES PISTES D'INDICES ACOUSTIQUES}

À la fin du test, dix auditeurs ont, dans leurs commentaires, mentionné des traits segmentaux et suprasegmentaux — attribués à des appartenances ethnolinguistiques plus que nationales. Les traits suprasegmentaux, afférents à la mélodie (aiguë, chantante) et au rythme (haché, rapide), étaient presque toujours attribués à l'accent wolof. Parmi les traits segmentaux, les sujets ont relevé une prononciation spécifique du [p] également attribuée à l'accent wolof, un déplacement des voyelles nasales attribué à l'accent bambara, un $/ \mathrm{J} /$ prononcé $[\mathrm{s}]$ et un $[\mathrm{r}]$ roulé attribués à l'accent mooré. La réalisation $[\mathrm{r}]$, trait le plus fréquemment cité, l'est cependant également pour d'autres accents.

Une analyse fine de la prononciation du /R/ a été menée sur les locuteurs ivoiriens, chez qui cette consonne peut être vocalisée ou élidée en position de coda, favorisant ainsi une structure CVCV (Boutin et Turcsan, 2009). Le /R/, également, est particulièrement affecté dans les groupes consonantiques, mais il l'est aussi en position intervocalique et même initiale. Le contexte phonologique ainsi que des facteurs sociolinguistiques concernant les locuteurs et les situations de parole ont été examinés, mais il semble qu'on ait affaire à un phénomène de variation libre: un même locuteur, dans un même énoncé et dans un même environnement phonologique, peut à quelques secondes d'intervalle alterner entre un [В] uvulaire perçu comme français et des variantes bien différentes. On sait que ce 
Tableau 5. Pourcentages par appartenance ethnolinguistique de /R/ apicaux, dorsaux ou labialisés/élidés.

\begin{tabular}{llllll}
\hline \hline \%/R/ & akan & bambara & sénoufo & mooré & wolof \\
\hline [r] apical & 34 & $\mathbf{9 5}$ & 55 & 73 & 59 \\
[b] dorsal & I6 & 0 & 0 & 6 & $\mathbf{2 3}$ \\
[w] labialisé ou élidé & $\mathbf{5 0}$ & 5 & 45 & I 8 & I 8 \\
\hline \hline
\end{tabular}

phonème, un des plus fréquents du français et de notre corpus, est polymorphe et combien il donne du fil à retordre aux phonéticiens (Autesserre et Chafcouloff, I999).

De façon analogue, les /R/ sous-jacents figurant dans les stimuli de notre expérience perceptive ont été annotés manuellement. Au nombre de 283, ils ont été classés en trois catégories: $[\mathrm{r}]$ apical, $[\mathrm{b}]$ dorsal, et [w] labialisé ou élidé. Le tableau 5 rapporte les résultats par appartenance ethnolinguistique — les résultats par pays concordent pour une large part. Les Akan (ou les Ivoiriens) sont ceux qui ont le plus de /R/ vocalisés ou élidés, et les Wolof (ou les Sénégalais) sont ceux qui produisent le plus de $/ \mathrm{R} /$ dorsaux. Les bambaraphones et les mooréphones sont ceux qui « roulent» le plus les /R/. Les Sénoufo leur ressemblent en ce qu'ils ne prononcent pas le «/R/ français» et ressemblent aux Akan en ce qu'ils l'affaiblissent souvent. Les chiffres correspondent assez bien à la conscience linguistique «naïve », avec peut-être une nuance à apporter pour les Sénégalais (wolophones). Ces derniers, même si la norme sénégalaise est le $/ \mathrm{r} / \mathrm{apical}$, produisent plus que leurs voisins de prononciations conformes à la norme hexagonale, rejoignant en cela des observations faites en wolof même, où des [в] à la française peuvent être utilisés (Moreau et Thiam, I995). Cette prononciation pouvant passer pour tubab, un jeu sociolinguistique extrêmement complexe est à l'œuvre.

Il semble également que les Wolof produisent des patrons prosodiques spécifiques. Dans notre corpus, les séquences « inconnue et tranquille » et «bataille politique » revenaient à chaque phrase lue (en position non-finale), et ont fait l'objet de divers commentaires. Aussi avons-nous mesuré la différence de fréquence fondamentale $\left(\Delta F_{\mathrm{o}}\right)$ entre les noyaux des syllabes finales et initiales de chacun de ces mots ([ny]-

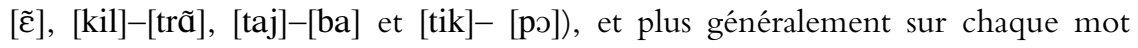
polysyllabique du texte lu par les locuteurs. Les valeurs de $F_{\mathrm{o}}$ ont été calculées toutes les io ms au moyen du logiciel PraAT ${ }^{4}$ avec les options par défaut, et moyennées par phonème. Les résultats, calculés en demi-tons, sont moyennés par appartenance ethnolinguistique dans le tableau 6. Moyennées par pays et sur les quatre mots, les valeurs de $\Delta F_{\mathrm{O}}$ sont de I, 3 demi-tons pour le Mali, I, 5 demi-tons pour le Burkina Faso, 2,6 demi-tons pour la Côte d'Ivoire et -I,7 demi-tons pour le Sénégal. Les Wolof (ou Sénégalais), en moyenne, sont donc les seuls à présenter des mesures négatives, correspondant à une mélodie descendante. Une telle intonation accompagnant ce qu'on peut interpréter comme des accents initiaux se retrouve

${ }^{4}$ http://www.praat.org 
Tableau 6. Différence de $F_{o}$ (en demi-tons) entre la syllabe finale et la syllabe initiale des mots inconnue, tranquille, bataille et politique, extraits de la phrase lue.

\begin{tabular}{llllll}
\hline \hline$\Delta F_{\mathrm{O}}$ (demi-tons) & akan & bambara & sénoufo & mooré & wolof \\
\hline Inconnue & 2,3 & $\mathrm{I}, 8$ & $\mathrm{I}, \mathrm{O}$ & $\mathrm{O}, 5$ & -3 \\
tranquille & $3, \mathrm{O}$ & $\mathrm{O}, 6$ & $\mathrm{I}, 3$ & $\mathrm{I}, 5$ & $-\mathrm{O}, 2$ \\
bataille & $\mathrm{I}, 2$ & $\mathrm{I}, 2$ & $\mathrm{I}, \mathrm{I}$ & 2,2 & $-\mathrm{I}, \mathrm{I}$ \\
politique & 5,2 & $\mathrm{O}, 6$ & $\mathrm{I}, 4$ & $\mathrm{I}, 8$ & $-2,2$ \\
\hline \hline
\end{tabular}
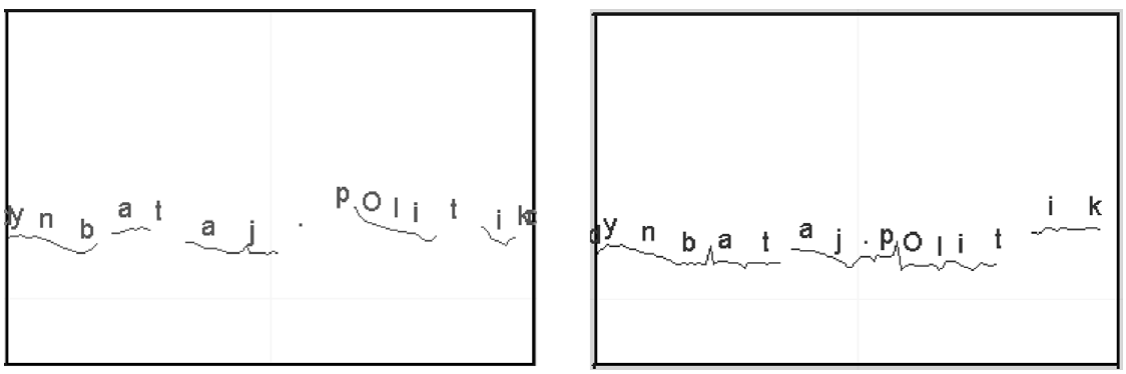

Figure 4. Courbes de $F_{\mathrm{O}}$ extraites par PRAAT pour la séquence une bataille politique lue par une locutrice sénégalaise wolof (à gauche) et une locutrice ivoirienne akan (à droite).

ailleurs, en lecture et en parole spontanée. Elle se confirme en moyennant les $\Delta F_{\mathrm{o}}$ sur tous les mots polysyllabiques du texte lu.

La figure 4 illustre les contours de $F_{\mathrm{O}}$ extraites par le logiciel PRAAT pour une locutrice sénégalaise wolof $(\mathrm{jB})$ et une locutrice ivoirienne akan (vC) lisant la séquence une bataille politique. On voit sur cet exemple que chez la locutrice sénégalaise (à gauche), dans le mot bataille le premier [a] est plus haut que le second et dans le mot politique la première voyelle est plus haute que la dernière. On a le patron inverse chez la locutrice ivoirienne (à droite).

\section{DISCUSSION ET PERSPECTIVES}

Ainsi, des résultats de cette expérience sur des échantillons de parole relativement courts, il ressort que la variation de type diastratique et diatopique (niveau d'études, appartenance ethnolinguistique et pays de résidence) est bien perçue et catégorisée par les auditeurs africains qui ont participé au test. Pour les jeunes locuteurs comme pour les locuteurs plus âgés, en lecture comme en parole spontanée, les résultats se sont montrés très robustes. Ils n'étayent pas, bien au contraire, l'hypothèse de l'émergence d'un « accent panafricain ».

Concernant l'âge des locuteurs, ces résultats differrent de ceux de tests réalisés en France métropolitaine: le degré d'accent perçu, comme le taux d'identification correcte, a tendance à augmenter avec l'âge des locuteurs sur le territoire français (Sobotta, 2006; Woehrling, 2009). Dans nos résultats, les «vieux » (plus de 47 ans) ne sont pas évalués avec plus d'accent que les «jeunes» (moins de 47 ans). Même si 
l'écart n'est significatif ni pour le degré d'accent ni pour le niveau d'études perçus, il contrecarre, a minima, une éventuelle hypothèse supposant un effacement des accents en cours. Ces premières constatations sont corroborées par le fait que, dans la tâche d'identification parmi 4 pays, de façon significative, les jeunes locuteurs laissent davantage transparaitre un accent national que les vieux locuteurs. Ce fait est pour nous parlant, allant dans le sens d'un changement du français après les Indépendances.

Les taux d'identification correcte parmi 5 catégories portant respectivement sur le niveau d'études et l'appartenance ethnolinguistique (respectivement $33 \%$ et $5 \mathrm{I} \%$ ) suggèrent que la variation de type diatopique est mieux identifiée que la variation de type diastratique. Nous avons vu également qu'il n'y a pas de relation simple entre niveau d'études et identification ethnogéographique.

L'impact du niveau d'études sur le degré d'accent perçu est particulièrement intéressant. Si les résultats manifestent une légère baisse du degré d'accent à mesure que le niveau d'études augmente, il faut attendre bac +3 pour voir ce degré d'accent diminuer notablement. D'un point de vue méthodologique, ces résultats montrent la pertinence d'une division fine du niveau d'études ainsi que la nécessité de tester des locuteurs d'un haut niveau d'études, souvent négligés dans les travaux sur le français en Afrique, ou bien considérés comme similaires à des locuteurs de niveau collège (Knutsen, 2007). D'un point de vue sociolinguistique, les résultats nous font supposer que l'école n'est pas le lieu d'un nivellement des accents: le processus de nivellement ne commence qu'après plusieurs années d'études supérieures. Cela est très certainement lié au fait que l'exposition au français international est peu fréquente hors de l'université et des milieux professionnels ouverts sur l'étranger. Cela est certainement lié aussi à l'attitude des locuteurs envers la langue, qui n'ont aucun motif à s'approprier le français international s'ils veulent s'insérer dans des milieux locaux.

Les résultats des tâches d'identification se sont dans l'ensemble montrés assez fidèles à la conscience linguistique auto-évaluée par les auditeurs. Ceux-ci se déclaraient confiants pour reconnaître la plupart des accents en présence. Tel a effectivement été le cas, alors que mise à l'épreuve de l'expérience, l'aptitude à identifier des accents régionaux et étrangers en français est bien souvent surestimée (Moreau, 2000; Boula de Mareüil et al., 2008).

De nombreuses études ont corroboré l'imprécision de l'identification/ caractérisation de ces accents (Bauvois, 1996; Léon et Léon, I997; Armstrong et Boughton, 1997; Fries et Deprez, 2003; Boughton, 2006; Woehrling, 2009). Nous n'avions que deux wolophones parmi nos auditeurs; cependant, l'accent wolof a été remarquablement bien identifié (à plus de $80 \%$ ). Il avait été également bien identifié par des auditeurs sénégalais (Moreau, 2000) - dans une étude perceptive dans laquelle l'échantillon de locuteurs ne comportait cependant pas de Maliens. Seuls les locuteurs sénoufo n'ont pas été bien identifiés, ce qui correspondait néanmoins à la conscience linguistique des auditeurs: avant le test, I 3 sur 20 ne se sentaient pas capables d'identifier un accent sénoufo, et après le test, I7 sur 20 déclaraient avoir répondu au hasard pour cette identification ethnolinguistique. 
Nous ne disposons d'aucune enquête perceptive faisant intervenir des auditeurs sénoufo, mais une étude récente (Lyche et Skattum, 20I0) a montré que des auditeurs maliens, parlant bambara et/ou fulfulde (peul), confondent également les accents bambara et sénoufo en français, alors que le Mali fait figure de pionnier dans la promotion des langues nationales. Les Sénoufo étant souvent bambaraphones, l'existence même d'un accent sénoufo reste, dans tous les cas, à prouver.

Dans les études antérieures comme dans celle-ci, la question reste entière de savoir si les confusions et les distinctions opérées relèvent de faits ethnolinguistiques ou nationaux. Nous ne prétendons pas, bien sûr, résoudre tous les problèmes autour de la notion de «statalismes ", c'est-à-dire de particularismes linguistiques plus ou moins emblématiques qui s'arrêteraient au passage d'une frontière politique (Frey, 2004). Il nous semble malgré tout que le présent travail fait un peu avancer le débat: les données et les problèmes méthodologiques sont brièvement résumés ici. Nous ne pensons pas que l'affichage du pays de provenance de quelques échantillons (et non de l'ethnie des locuteurs), lors de la phase de familiarisation au début de notre expérience, aient pu influencer les résultats.

Il faut d'abord tenir compte des représentations des accents nationaux en Afrique de l'Ouest: l'accent ivoirien est, par défaut et pour des raisons historiques, l'accent agni (kwa/akan), l'accent burkinabè est identifié avec celui des Mossi, l'accent malien avec celui des Bambara et l'accent sénégalais avec celui des Wolof. Dans les réponses de nos auditeurs, nous observons des associations quasiment fixes entre pays et ethnie (dans plus de 90\% des cas). Par ailleurs, comment s'assurer que les auditeurs ne sont pas tributaires de certaines représentations sociales et, partant, d'associations qui biaisent une réelle identification géographique? Nous nous sommes, par exemple, demandés si des niveaux d'études bas et hauts allaient de pair avec l'identification à certains pays ou groupes ethnolinguistiques. Cependant, nous l'avons dit, le lien est faible entre l'évaluation du degré d'accent ou du niveau d'études et l'identification de l'appartenance ethnolinguistique ou du pays des locuteurs. Quelques éléments peuvent être tirés d'une étude telle que celleci, sur un détachement d'accents plutôt nationaux ou plutôt ethnolinguistiques. Même si le nombre réduit de locuteurs recrutés par pays ne permettait pas de représenter beaucoup de groupes ethnolinguistiques, seul le Sénégal n'avait qu'une ethnie représentée; les autres pays en comptaient deux ou trois. Les résultats des identifications, parmi 4 pays et 5 ethnies, comme leur interprétation, ne sont pas directement comparables. Mais pour chaque pays, l'identification a été correcte dans la majorité des cas, alors que le groupe sénoufo, réparti sur trois pays, a été mal identifié. Ces faits sont sans doute le reflet de ce que le français n'est pas appris comme une langue étrangère mais comme une langue qui fait l'objet d'une appropriation communautaire d'envergure nationale, avec des identités en train de se construire au-delà des identités ethnolinguistiques. Le rôle des médias dans cette insertion du français dans les nations, de la radio, des talk-shows télévisés, n'est pas à écarter. D'autres études sont requises: pour faire la part entre le national et l'ethnolinguistique, il faudrait inclure un plus grand nombre de locuteurs d'une même ethnie répartie sur plusieurs pays. 
Arriver à définir ces accents, les caractériser avec précision, est une tout autre affaire, bien sûr plus difficile. Reconnaitre un accent, en effet, fait appel à des ressources cognitives et à des routines variées, conscientes et inconscientes. Les Ivoiriens «ne prononcent pas les ' $r$ ' », à ce qu'on dit, et nos mesures le corroborent. Il reste que les différences perçues entre les variétés de français étudiées ne sont pas réductibles à ce seul trait. Pour séparer les locuteurs sur la base de leur appartenance géo/ethnolinguistique, d'autres traits plus ou moins bien documentés (Cissé, 2006) sont à déterminer, au-delà des lieux communs souvent réversibles (une variété $X$ est décrite comme chantante par les locuteurs d'une variété $Y$ et vice versa). Les Wolof n'ont pas les contraintes que connaissent les langues à tons, mais peuvent produire des accents initiaux et/ou des patrons mélodiques descendants, également en français. Le lien avec le rythme est une piste à creuser. En tout état de cause, des études plus approfondies sont nécessaires pour cerner les indices acoustiques mobilisés pour distinguer les différents accents ouest-africains. L'analyse envisagée pourra porter sur davantage de données; quant à l'approche globale, elle mérite d'être étendue à des auditeurs et des locuteurs d'autres origines.

Adresse pour correspondance:

Phillippe Boula de Mareüil

LIMSI-CNRS

BP 133

F-91403 Orsay CEDEX

France

e-mail:Philippe.Boula.de.Mareuil@limsi.fr

\section{REFERENCES}

Armstrong, N. et Boughton, Z. (I997). Identification and evaluation responses to a French accent: some results and issues of methodology. Revue PArole, 5-6: 27-60.

Autesserre, D. et Chafcouloff, M. (I999). Étude expérimentale du rôle de l'organisation syllabique dans la prédiction des variantes de /R/ en français. $2^{\text {es }}$ Journées d'Études Linguistiques, Nantes, pp. I 54-159.

Bauvois, C. (1996). Parle-moi, et je te dirai peut-être d'où tu es. Revue de Phonétique Appliquée, I2 I: 291-309.

Boughton, Z. (2006). When perception isn't reality: accent identification and perceptual dialectology in French. Journal of French Language Studies, I6: 277-304.

Boula de Mareüil, P., Vieru-Dimulescu, B., Woehrling, C., Adda-Decker, M. (2008). Accents étrangers et régionaux en français. Caractérisation et identification. Traitement Automatique des Langues, 49, 3: I35-I62.

Boutin, B. A. et Turcsan, G. (2009). La prononciation du français en Afrique: la Côte d'Ivoire. Dans: J. Durand, B. Laks et C. Lyche (dir.), Phonologie, variation et accents $d u$ français. Paris: Hermès, pp. I 3 I-I 52.

Boutin, B. A. et Prignitz, G. (20IO). Conversation à Ouagadougou (Burkina Faso): parenté à plaisanterie entre Gurma et Yatenga. Dans: S. Detey, J. Durand, B. Laks et 
C. Lyche (dir.), Le français parlé contemporain dans ses variétés. Ressources pour l'étude du français. Paris: Ophrys, pp. 259-272.

Boutin, B. A. et Gueye, G. M. (à paraitre). French in Senegal after three centuries: A phonological study. Dans: R. Gess, C. Lyche et T. Meisenburg (dir.), Phonological Variation in French: Illustrations from Three Continents. Amsterdam/Philadelphia: John Benjamins.

Canut, C. (1996). Dynamique linguistique au Mali, Paris: Didier Érudition.

Canut, C. (1997). Imaginaire linguistique en Afrique, Paris: L'Harmattan.

Cissé, M. (2005). Langues, État et société au Sénégal. Sudlangues, 5: 99-I33.

Cissé, M. T. (2006). Problèmes de phonétique et de phonologie en wolof. SudLangues, 6: $23-62$.

Clopper, C. G., et Bradlow, A. R. (2009). Free classification of American English dialects by native and non-native listeners. Journal of Phonetics, 37, 4: 436$45 \mathrm{I}$.

Durand, J., Laks, B., Lyche, C. (2002). La phonologie du français contemporain: usages, variétés et structure. Dans: C. Pusch et W. Raible (eds.), Romanistische Korpuslinguistik - Korpora und gesprochene Sprache, Tübingen: Gunter Narr Verlag, pp. 93-Io6.

Durand, J., Laks, B., Lyche, C. (2005). Un corpus numérisé pour la phonologie du français. Dans: G. Williams (dir.), La linguistique de corpus, Rennes: Presses Universitaires de Rennes, pp. 205-2I7.

Frey, C. (2004). Particularismes lexicaux et variétés de français en Afrique francophone: autour des frontières. Glottopol, 4: I 36-I49.

Fries, S. et Deprez, C. (2003). L'accent étranger: identification et traitement social en France et aux États-Unis. Cahiers du français contemporain, 8: 89-105.

Kuiper, L. (2005). Perception is reality: Parisian and Provençal perceptions of regional varieties of French. Journal of Sociolinguistics, 9/ 1: 28-52.

Knutsen, A. M. (2007). Le français à Abidjan (Côte d'Ivoire). Vers une analyse multidimensionnelle de la variation. Revue du Réseau des Observatoires du Français Contemporain en Afrique, 22: I27-I 59 .

Labov, W. (I972). Sociolinguistic patterns, Philadelphia: University of Pennsylvania Press.

Lafontaine, D. (1986). Le parti pris des mots. Normes et attitudes linguistiques, Bruxelles: Pierre Mardaga.

Lambert, W. E. (1972). Language, psychology and culture, Stanford: Stanford University Press.

Léon, P. et Léon, M. (1997). La prononciation du français, Paris: Fernand Nathan.

Lyche, C. et Skattum, I. (20I0). Le rôle de la Li dans le français du Mali: une étude perceptive. $2^{e}$ Congrès Mondial de Linguistique Française, Nouvelle Orléans, pp. I9I $3-$ I926.

Moreau, M.-L. (2000). Le français d'Afrique: Phénomènes d'interférence ou de socialisation? Considérations méthodologiques. Dans: M.-A. Hintze, T. Pooley et A. Judge (eds), French accents: phonological and sociolinguistic perspectives, Londres: AFLS/CiLT, pp. 288-304.

Moreau, M.-L. et Thiam, N. (I995). "Comment je reconnais les variétés du wolof" Le discours des adolescents sur les variétés régionales et ethniques du wolof. Sciences et Techniques du Langage, I: 49-53.

Ploog, K. (2002). Le français à Abidjan. Pour une approche syntaxique du non-standard, Paris: Éditions du CNRS. 
Évaluation et identification perceptives d'accents ouest-africains en français

Preston, D. R. (1989). Perceptual Dialectology. Dordrecht: Foris.

Scherfer, P. (2000). La notion de conscience linguistique. Dans: G. Holtzer et M. Wendt (éd.), Didactique comparée des langues et études terminologiques: Interculturel-StratégiesConscience langagière, Berne: Peter Lang, pp. I69-i 84.

Sobotta, E. (2006). Phonologie et migration - Aveyronnais et Guadeloupéens à Paris, thèse de doctorat des universités Paris X-Nanterre et LMU de Munich.

Tymian, J., N'Guessan, J. K., Loucou, J.-N. (2003). Dictionnaire baoulé-français, Abidjan: Nouvelles Éditions Ivoiriennes.

Woehrling, C. (2008). Accents régionaux en français: perception, analyse et modélisation à partir de grands corpus, thèse de doctorat de l'université Paris-Sud XI, Orsay. 